\title{
The CTB1 Gene Encoding a Fungal Polyketide Synthase Is Required for Cercosporin Biosynthesis and Fungal Virulence of Cercospora nicotianae
}

\author{
Mathias Choquer, ${ }^{1}$ Katherine L. Dekkers, ${ }^{1}$ Hui-Qin Chen, ${ }^{1}$ Lihua Cao, ${ }^{1}$ Peter P. Ueng, ${ }^{2}$ \\ Margaret E. Daub, ${ }^{3}$ and Kuang-Ren Chung ${ }^{1,4}$ \\ ${ }^{1}$ Citrus Research and Education Center, Institute of Food and Agricultural Sciences (IFAS), University of Florida, 700 \\ Experiment Station Road, Lake Alfred 33850, U.S.A.; ${ }^{2}$ Molecular Plant Pathology Lab., United States Department of \\ Agriculture-Agricultural Research Service, BARC-West, 10300 Baltimore Ave., Beltsville, MD 20705, U.S.A.; ${ }^{3}$ Departments \\ of Botany and Plant Pathology, North Carolina State University, Raleigh 27695, U.S.A.; and ${ }^{4}$ Department of Plant Pathology, \\ IFAS, University of Florida, Gainesville 32611, U.S.A.
}

Submitted 22 October 2004. Accepted 10 January 2005.

Cercosporin is a light-activated, non-host-selective toxin produced by many Cercospora fungal species. In this study, a polyketide synthase gene (CTB1) was functionally identified and molecularly characterized to play a key role in cercosporin biosynthesis by Cercospora nicotianae. We also provide conclusive evidence to confirm the crucial role of cercosporin in fungal pathogenesis. $C T B 1$ encoded a polypeptide with a deduced length of 2,196 amino acids containing a keto synthase (KS), an acyltransferase (AT), a thioesterase/claisen cyclase (TE/CYC), and two acyl carrier protein (ACP) domains, and had high levels of similarity to many fungal type I polyketide synthases. Expression of a 6.8 -kb CTB1 transcript was highly regulated by light and medium composition, consistent with the conditions required for cercosporin biosynthesis in cultures. Targeted disruption of $C T B 1$ resulted in the loss of both $C T B 1$ transcript and cercosporin biosynthesis in $C$. nicotianae. The $c t b 1$-null mutants incited fewer necrotic lesions on inoculated tobacco leaves compared with the wild type. Complementation of $c t b 1$-null mutants with a full-length $C T B 1$ clone restored wild-type levels of cercosporin production as well as the ability to induce lesions on tobacco. Thus, we have demonstrated conclusively that cercosporin is synthesized via a polyketide pathway, and cercosporin is an important virulence factor in $C$. nicotianae. The results also suggest that strategies that avoid the toxicity of cercosporin will be useful in reduction of disease incidence caused by Cercospora spp.

Additional keywords: gene disruption, pathogenicity, perylenequinone.

Cercospora spp. are a highly successful group of fungal pathogens causing disease in more than 100 plant species, such as soybean, sugar beet, coffee, rice, corn, peanut, and tobacco (Daub and Ehrenshaft 2000). Cercospora spp. cause major economic problems because of their worldwide distribution, wide host range, and low levels of resistance in many host plants. Many Cercospora spp. produce a non-host-specific toxin, cercosporin (1,12-bis-(2-hydroxypropyl)-2,11-dimethoxy-6,7methylenedioxy-4,9-dihydroxyperylene-3,10-quinone), which

Corresponding author: K.-R. Chung; Telephone: (863) 956-1151 ext. 1369; Fax: (863) 956-4631; E-mail: krchung@crec.ifas.ufl.edu has been considered to be an important virulence factor (Upchurch et al. 1991). Cercosporin is a light-activated photosensitizing toxin that, upon illumination, interacts with molecular oxygen to produce both superoxide radicals and singlet oxygen (Daub and Ehrenshaft 2000; Daub and Hangarter 1983). These activated oxygen species cause peroxidation of cell membrane lipids and breakdown of the host plasma membrane, resulting in electrolytic leakage, a decrease in membrane permeability, and, eventually, cell death (Daub 1982a). Nutrients leaking from host cells support pathogen growth and sporulation in the host (Daub and Ehrenshaft 2000).

Cercosporin first was isolated from a soybean pathogen, Cercospora kikuchii (Kuyama and Tamura 1957), and its toxicity via photosensitizing activity to mice, bacteria, several fungi, and host plants has been established to be light and oxygen dependent (Daub 1982b; Macri and Vianello 1979; Yamazaki et al. 1975). Despite the universal toxicity of cercosporin, Cercospora spp. apparently have evolved sophisticated mechanisms associated with transient reduction of cercosporin, singlet oxygen quenching, toxin export, and other unknown functions that allow them to tolerate cercosporin toxicity (Callahan et al. 1999; Chung et al. 2003b; Daub et al. 1992, 2000; Ehrenshaft et al. 1998, 1999). Production of cercosporin is highly affected by environmental factors (e.g., nutrient conditions, light, temperature, and cultural age) and signal transduction involving calcium or calmodulin and MAP kinase pathways (Chung 2003; Daub and Ehrenshaft 2000; Ehrenshaft and Upchurch 1991, 1993; Jenns et al. 1989; Shim and Dunkle 2003). However, little progress has been made toward understanding the biosynthetic pathway of cercosporin. Early studies using nuclear magnetic resonance and mass-spectrometric analyses of products derived from fungal culture fed with ${ }^{14} \mathrm{C}$-labeled acetate suggested that cercosporin is produced via a polyketide pathway by the condensation of acetate and malonate subunits (Okubo et al. 1975). No chemical intermediates or enzymes have ever been identified for the cercosporin biosynthetic pathway, however.

Cercosporin has been suggested to play a crucial role in virulence of Cercospora spp. for many years. Light intensity is correlated with both disease severity and toxin action (Calpouzos and Stalknecht 1967; Daub 1982b). Cercosporin has been isolated from affected tissues, and application of toxin alone can produce similar symptoms on host plants (Balis and Payne 1971; Fajola 1978; Upchurch et al. 1991). Non-toxinproducing mutants of $C$. kikuchii derived from UV mutagene- 
sis failed to cause disease symptoms in soybean plants (Upchurch et al. 1991). Furthermore, disruption of a CFP gene encoding a facilitator protein in $C$. kikuchii (Callahan et al. 1999) and a $C Z K 3$ gene encoding a MAP kinase kinase kinase homolog in C. zeae-maydis (Shim and Dunkle 2003) resulted in mutants that were defective in cercosporin biosynthesis and that also exhibited lower virulence on soybean and maize, respectively. Disruption of $C F P$ and $C Z K 3$ genes resulted in pleiotrophic phenotypes; therefore, the pathological roles of cercosporin in symptom development and fungal pathogenesis remain undefined at molecular levels.

In previous studies using the restriction enzyme-mediated integration (REMI) approach, we identified several groups of mutants of $C$. nicotianae that are altered in cercosporin production (Chung et al. 2003c). Sequence analysis of a flanking DNA fragment in the rescued plasmid clone (pCTB1) recovered from one of the cercosporin-deficient mutants revealed a sequence with strong amino acid similarities to many fungal polyketide synthases (PKSs) in the databases. We partially characterized the clone as CTB1 (for cercosporin toxin biosynthesis) and proposed that it encoded a fungal PKS responsible for cercosporin production (Chung et al. 2003c). In this article, we describe the complete cloning, regulated expression, and functional characterization of the CTB1 gene and, using $c t b 1$ disrupted mutants, unambiguously demonstrate its involvement in cercosporin biosynthesis and requirement for fungal pathogenesis in C. nicotianae.

\section{RESULTS}

\section{Molecular cloning and characterization of $C T B 1$ gene encoding a fungal PKS.}

Partial CTB1 sequences showing amino acid similarities to various fungal PKSs were identified first from a 1.6-kb flanking region rescued from a cercosporin-deficient REMI mutant (ctb1) (Chung et al. 2003c). The full-length CTB1 gene subsequently was obtained by polymerase chain reaction (PCR) with two inverse primers using strategies outlined in Figure 1. The primers (pks5/pks6 and 1209L/1209R) were designed based on the sequences in the 1.6-kb flanking DNA and were used for PCR amplification from EcoRV- and NcoIdigested and self-ligated genomic DNA pools. Two overlapping fragments were amplified and cloned into the pGEM-T easy vector, resulting in plasmids pKS-8 and pCNA1, respectively (Fig. 1). The cDNA fragments of the CTB1 were cloned collectively and sequenced as four overlapping clones, pXOC2, pXOC7, pXOC10, and pTAR3 (Fig. 1). Analysis of the combined sequences derived from genomic clones and the cDNA identified a 6,591-bp open reading frame (ORF) and the presence of eight small introns ranging from 48 to $74 \mathrm{bp}$ in size. Seven of the eight introns (except intron VI) had characteristic 5'-XX/GT and AG/XX-3' splicing junctions commonly found in filamentous fungi. Six of them also contain an internal lariat consensus sequences CT(A/G) A (T/C) (Radford and Parish 1997). Sequence analysis of 520 nucleotides upstream of the ATG start codon revealed that the promoter region of $C T B 1$ contains one putative TATA box and three putative CAAT elements (data not shown). The predicted start codon and flanking sequences of $C T B 1$ also have the eukaryotic translation initiation consensus sequence [CA(C/A)(A/C)ATGGC] (Kozak 1984). Using 5' and 3' RACE, putative transcriptional initiation sites were mapped to 101 and $172 \mathrm{bp}$ upstream of the predicted ATG translational start codon, and the transcriptional termination site was localized $57 \mathrm{bp}$ downstream of the translational stop codon. The CTB1 sequence was deposited in the GenBank database under accession number AY649543.
The CTBl gene was predicted to encode a polypeptide of 2,196 amino acids (aa) rich in alanine (11.2\% overall). The CTB1 protein had a predicted molecular mass of $236.7 \mathrm{kDa}$ and a predicted pI of 5.6. BlastP search (Altschul et al. 1997) of the National Center for Biotechnology Information (NCBI) protein database revealed that the predicted 2,196-aa translation product of $C T B 1$ has 35 to $40 \%$ identity and 50 to $60 \%$ similarity to several type I fungal PKSs. Analysis of the predicted translational product of the $C$. nicotianae CTB1 gene revealed the presence of a keto synthase (KS), an acyltransferase (AT), a thioesterase/ claisen cyclase (TE/CYC), and two acyl carrier protein (ACP, phosphopantotheine attachment site) domains (Fig. 2A). Comparisons of the individual domains within various fungal PKSs are shown in Figure 2B. In addition to the KS, AT, TE/CYC, and ACP domains, comparison of the $\mathrm{N}$-terminal sequences revealed that CTB1 has a conserved AFGDQT sequences similar to those of fungal type-I PKSs. No conserved amino acid sequences similar to ketoreductase (KR), dehydratase (DH), and enoyl reductase (ER) domains were found in CTB1.

\section{Gene disruption and genetic complementation.}

The function of the CTB1 in C. nicotianae was determined using a gene disruption strategy. A disruption vector pCTB115 containing a hygromycin-resistance gene $(H Y G)$ cassette was developed and used for $C T B 1$ gene replacement. To facilitate

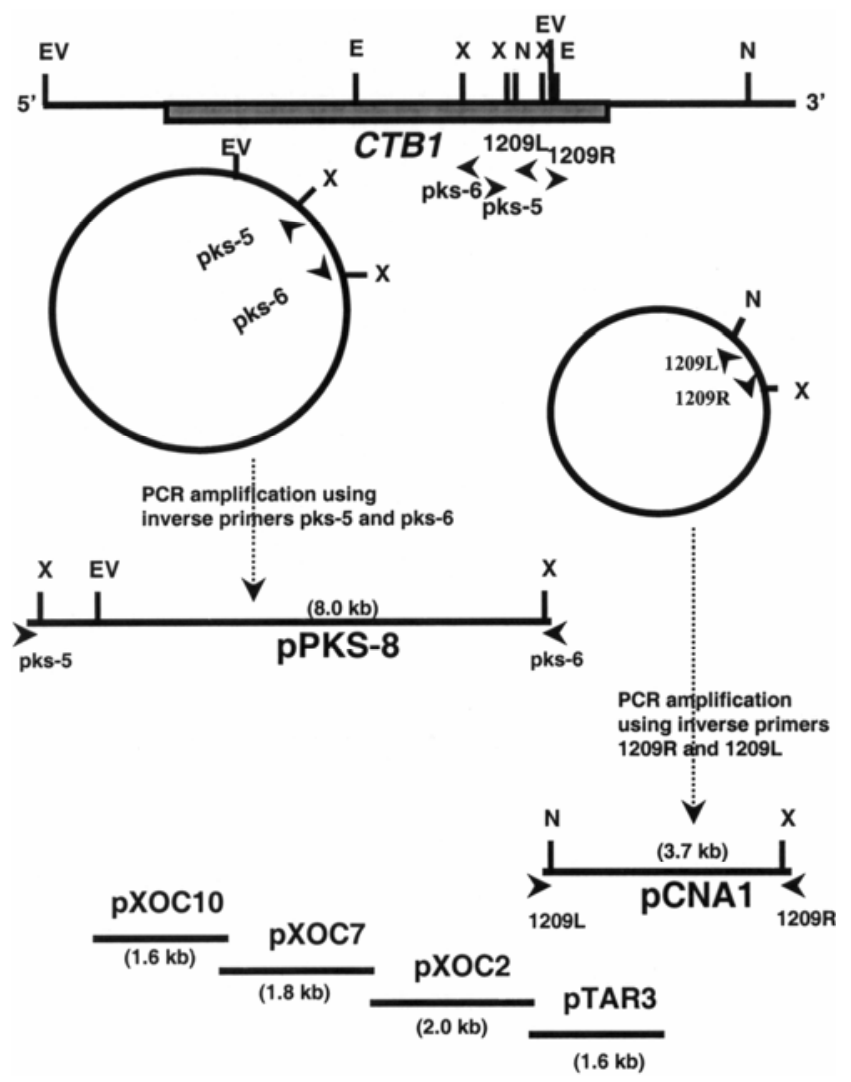

Fig. 1. Restriction map of the Cercospora nicotianae CTB1 gene and schematic describing the strategy to clone the $5^{\prime}$ and $3^{\prime}$ regions. Arrowheads indicate relative positions of primers (pks5/pks6 and 1209L/1209R) used for polymerase chain reaction (PCR) amplification. DNA fragments amplified from EcoRV- and NcoI-digested, self-ligated genomic pools were cloned into a pGEM-T vector to yield pPKS-8 and pCNA1, respectively. Four overlapping cDNA fragments encompassing the entire CTB1 gene as well as the $5^{\prime}$ and $3^{\prime}$ nontranslation regions were obtained from reverse-transcriptase PCR, and cloned to result in pXOC10, pXOC7, pXOC2, and pTAR3 for sequencing analysis. Restriction enzymes used were: $\mathrm{E}=$ $E c o R I, E V=E c o R V, N=N c o I$, and $\mathrm{X}=X h o I$. Note: drawing is not to scale. Details on cloning are indicated in the methods section. 
A

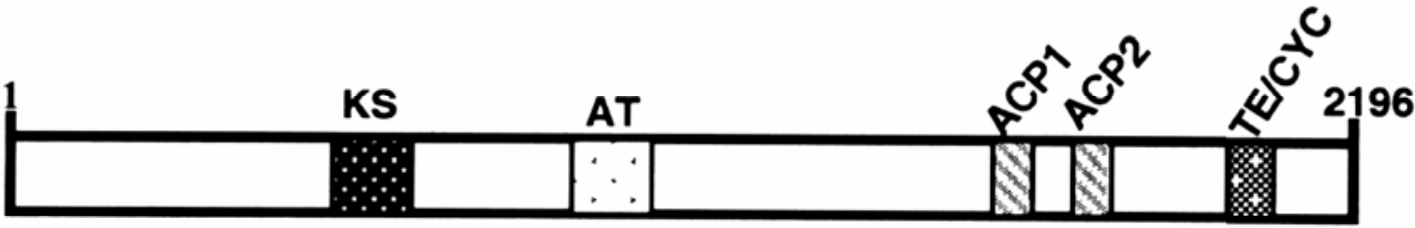

B

$\beta$-Ketoacyl Synthase (KS) domain (acyl binding cysteine)

CTB1 GRVNYFHKFSGPSYTIDTACSSSLAALHMACNALWRGEVDTAIVGGT

WDPKS1 GXINYFFKFSGPXFSVDTACSSSLAAMNVAITSLRANECDTVFTGGA

WA GRINYYFKFSGPSVSVDTACSSSLAAIHLACNSIWRNDCDTAITGGV

PKSL GRINFCFEFAGPSYTNDTACSSSLAAIHLACNSLWRGDCDTAVAGGT

STCA GRINFCFEFSGPSYSNDTACSSSLAAIHLACNSLWRGDCDTAVAGGT

BfPKS13 GRINYHFGFSGPSYSVDTACSSSMAAINLAVTSLRAGDCDTVFAGGM

ALB1 GRINYYFKFSGPSVSVDTACSSSLAAIHLACNAIWRNDCDTAISGGV

Consensus DXACESSL

Acyl Transferase (AT) domain (pantotheine binding serine)

CTB1 QMALYRLWKSLGVQAKAVVGHSLGEYAALYAAGVLSQSDTLYLVGRRA

WDPKS1 QMALTHLWSTWGIQPSVVIGHSLGEYAALQAAGVLSIADTIYLVGKRA

WA QMALSSFWASLGITPSFVLGHSLGDFAAMNAAGVLSTSDTIYACGRRA

PKSL QMALTNLMTSFGIRPDVTVGHSLGE FAALYAAGVLSASDVVYLVGQRA

STCA EMALGNLLGSFGLKPSAVIGHSLGEYAALYISGVLSAADTLYLVGMRA

BfPKS13 EIAVAALWQSWGIEPSAVVGHSLGEYAALQVAGVISTHDAIALVGNRA

ALB1 QMALVDYWKGLGVTPAFVLGHSLGDYAALNSAGVLSTSDTIYLCGRRA

Consensus GHSXG

Acyl carrier protein ( $A C P$ ) domain 1 (phosphopantotheine binding serine)

CTB1

WDPKS1

LEIVSEE IGMPIDELKDDIDFTDAGLDSLLSLVISSRMRDQLGIEFES

FE I IAKE IDVDQSELNDDIQWADMGVDSLMSLTISGKFREDLDLEVXS

WA LNILASEVGLSESDMSDDLVFADYGVDSLLSLTVTGKYREELNLDMDS MRVVSEESGIALEELTDDSNFADMGIDSLSSMVIGSRFREDLGLDLGP

PKSL

STCA LSVISEESGVALGELTADTNFADIGIDSLSSMVIGSRLREDLGLELGA

BfPKS13

ALB1 LAIMAEE IGCDVAELAGPMRFSDMGVDSLLGLSISGRFREDLE IDFQS LE ILAAEVGLSEAEMTDSLNFADYGVDSLLSLTVTGRYREELNLDLES

Consensus GXD $\underline{L} L$

Acyl carrier protein (ACP) domain 2 (phosphopantotheine binding serine)

CTB1

SEESGLTDEELTDDTSFADVGVDSLMSLVITSRLRDELDIDFPDRALFE

WDPKS1

WA AQEMEMDLAE ITDXTDLSNLGMDSLMALTVLGKLREDHDIDLDPTILAD ADE IGVSADE IKSDENLNELGMDSLLSLTVLGKIRE SLDMDLPGEFFIE

PKSL

STCA

BfPKS13 SEESGIALEELTDDSNFADMGIDSLSSMVIGSRFREDLGLDLGPEFSLF SEESGVALDELSAETVFADIGIDSLSSMVITSRFREDLGMSLDSSFNLF AEEMGVEMEE ITDNTDLATMGMD SLMSLSILGALREKTGLNLSSELLVE

ALB1 AEE IGVSVGDIDPSDNLPEMGMD SLLSLTVLGRIRETLGMDLPAEFFLE

Consensus GXD $\underline{S} L$

Thioesterase

CTB1

WDPKS1

WA1

PKSL

STCA

BfPKS13

ALB1

Consensus

se (CYC) motif

(TE) / Claisen cyclase (CYC) motif

YLEKVLXRQPNGPY ILXGWSASGVFAYXITXQLXDLQXLHPDKNYTVEK YLAE IRRRQPTGPYNLGGWSAGGICAYDAARKLVLQQGE IVETLLLLDT FCNE IRRRQPRGPYHLGGWSSGGAFAYVVAEALVNQGEEVHS-LIIIDAP FCNE IKRRQPEGPYHLGGWSSGGAFAYVTAEALINAGNEVHS-LI I IDAP YLAE IQRRQPHGPYLLGGWSAGGVLAYECTRQLIAKGEKVEK-LVLIDSP YLAE IRRRQPKGPYSFGGWSAGGICAFDAARQLILEEGEEVERLLLLDSP GWSAGG

Fig. 2. A, Physical map of CTB1 protein (GenBank accession number AY649543) with a keto synthase (KS), an acyltransferase (AT), a thioesterase/claisen cyclase (TE/CYC), and two acyl carrier protein (ACP, phosphopantotheine attachment site) domains in Cercospora nicotianae. B, Alignments of CTB1 with conserved active sites of other fungal polyketide synthases (PKSs). Amino acid sequences shown are as follows: Exophiala dermatitidis WDPKS1 (accession number AAD31436), Emericella nidulans WA (Q03149), Aspergillus parasiticus PKSL (Q12053), E. nidulans STCA (Q12397), Botryotinia fuckeliana BfPKS13 (AAR90249), and A. fumigatus ALB1 (AAC39471). Amino acid identities to those of CTB1 in C. nicotianae are shaded. Conserved amino acids for all peptides are shown under each alignment, and the active amino acids are underlined. 
gene replacement, DNA fragments containing a split hygromycin phosphotransferase B gene $(H Y G)$ marker $\left(5^{\prime} C T B 1\right.$ fused with $3^{\prime} H Y G$ fragment and 5' $H Y G$ fused with $3^{\prime} C T B 1$ fragment) were amplified from pCTB115 (Fig. 3A), and the PCR products were directly transformed into the wild-type strain. Because cercosporin is a red pigment, toxin-deficient mutants can be detected easily by screening for lack of red pigmentation in the medium. Fungal transformants were first selected on a medium supplemented with hygromycin and then tested for cercosporin production by plating on thin potato dextrose agar (PDA) plates. The wild-type isolate displayed dark red color due to secretion and accumulation of cercosporin around hyphae. Transformants that lacked red pigmentation were identified as cercosporin-deficient mutants. In all, 16 of 41 transformants displayed complete lack of visible cercosporin production.

Southern blot analysis of genomic DNA from three putative disruption mutants ( $\Delta$ ctb1-T1, T11, and T18) showed that they all had an integrated fragment at the $C T B 1$ site in the genome (Fig. 3B). Fungal genomic DNA was digested with NcoI and $E c o \mathrm{RV}$ and hybridized to a $C T B 1$ gene probe generated using

\section{A Split-hygromycin phosphotransferase B gene (HYG) marker}

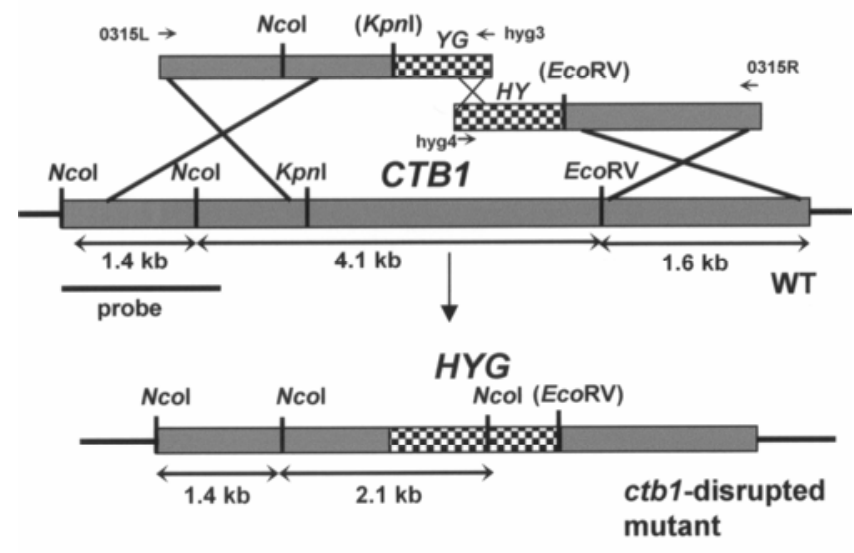

B
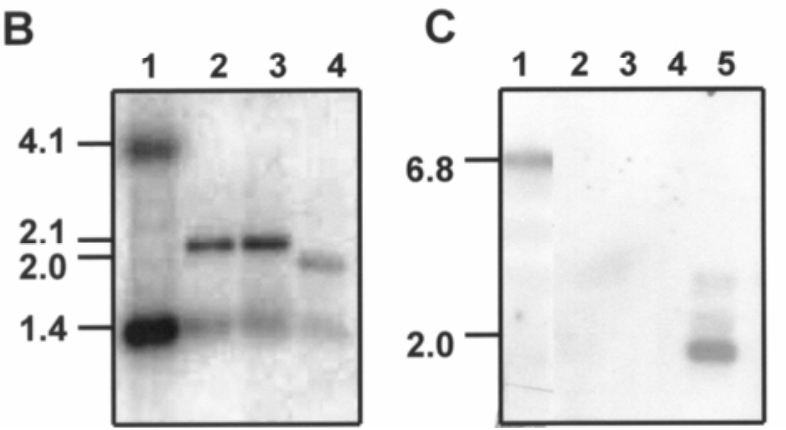

Fig. 3. Targeted gene disruption of $C T B 1$ using a split-marker strategy in Cercospora nicotianae. A, Restriction maps of the CTB1 gene in the wild type (WT) and $c t b 1$-disrupted fungal genome. Two truncated CTB1 fragments fused with an overlapped $H Y / Y G$ ( $H Y G=$ hygromycin resistance gene cassette) were obtained by polymerase chain reaction (PCR) amplification as described in the text. Oligonucleotide primers (0315L/hyg3, 0315R/hyg4) used for PCR amplification also are shown. Note: drawing is not to scale. B, Southern blot analysis of genomic DNA from the wild type (lane 1) and three $c t b 1$ disruption mutants: $\Delta$ ctb1-T1 (lane 2), $\Delta$ ctb1-T11 (lane 3), and $\Delta$ ctb1-T18 (lane 4). Fungal DNA was digested with $N c o$ I and EcoRV, electrophoresed, blotted onto a nylon membrane, and hybridized with a $C T B 1$-specific probe. The membrane was washed at high stringency. Sizes of hybridizing bands are indicated in kilobase pairs $(\mathrm{kb})$. Band patterns indicate disruption of the $C T B 1$ gene, as described in the text. C, Northern blot analysis of total RNA from the wild type (lane 1) and four $c t b 1$ disruption mutants: $\Delta$ ctb1-T1 (lane 2), $\Delta$ ctb1T11 (lane 3), $\Delta$ ctb1-T6 (lane 4), and $\Delta$ ctb1-T7 (lane 5).
PCR. Both the wild-type strain and the putative $c t b 1$-disruptants contained a 1.4-kb NcoI hybridizing band in the genomic DNA (Fig. 3B, lanes 1 to 4). The probe also hybridized to a 4.1-kb NcoI-EcoRV fragment in the wild-type strain (lane 1). Because an NcoI restriction enzyme site is present in the $H Y G$ gene cassette, hybridization of the genomic DNA from the ctb1-disruptants failed to detect the 4.1-kb hybridizing band, but detected a 2.1-kb NcoI-hybridizing band (lanes 2 and 3). The 2.0-kb hybridizing band in the $\Delta \mathrm{ctb} 1-\mathrm{T} 18$ mutant (lane 4) was smaller than expected, likely due to a small deletion caused by the double crossover recombination. Nevertheless, these results indicate that $C T B 1$ was replaced successfully with the marker gene. Northern blot analysis of total RNA from four $c t b 1$-disruptants $(\Delta c t b 1-\mathrm{T} 1, \mathrm{~T} 11, \mathrm{~T} 6$, and $\mathrm{T} 7)$ using a $C T B 1$ gene probe failed to detect the $6.8-\mathrm{kb}$ transcript of $C T B 1$ (Fig. 3C), strongly indicating that these transformants are $c t b 1$-null mutants. Due to the orientation of the disruption construct, a truncated transcript (less than $2 \mathrm{~kb}$ ) was detected in the $\Delta$ ctb1-T7 mutant (Fig. 3C, lane 5).

Genetic complementation was performed to further verify the role of $C T B 1$ in cercosporin biosynthesis. Protoplasts prepared from two $c t b 1$-null mutants ( $\Delta$ ctb-T1 and T19) were transformed with plasmid pCTB117 containing the full-length CTB1 gene as well as its upstream and downstream flanking sequences. A total of $25 \Delta$ ctb-T1 and of $34 \Delta$ ctb-T19 transformants exhibited wild-type levels of cercosporin production after complementation with CTBI (Table 1), confirming the involvement of $C T B 1$ in cercosporin biosynthesis.

Cercosporin production by the $c t b l$ disruptants and disruptants complemented with $C T B 1$ was measured and compared with that of the wild type. Mycelial plugs were extracted with $5 \mathrm{~N} \mathrm{KOH}$ and the amounts of cercosporin in the extract quantified by measuring absorbance of the extract at $480 \mathrm{~nm}$ (Jenns et al. 1989) (Table 1). Agar plugs soaked with $5 \mathrm{~N} \mathrm{KOH}$ resulted in light yellow pigment, producing absorbance values of approximately 3 to $6 \mathrm{nmoles} / \mathrm{plug}$. The wild-type and the complemented disruption mutants produced large amounts of cercosporin (122 to 136 nmoles/plug). Very low absorbance values,

Table 1. Production of cercosporin and necrotic lesions on tobacco by the Cercospora nicotianae wild-type strain ( $\mathrm{CnA})$, the $\operatorname{crg} 1$-null mutant (205C3), the $c t b 1$-null mutants ( $\Delta$ ctb1-T), and the CTB1-complemented disruption strains (T1R18 and T19R5)

\begin{tabular}{lcc}
\hline Isolates & $\begin{array}{c}\text { Cercosporin production } \\
(\text { nmole/agar plug) }\end{array}$ & $\begin{array}{c}\text { Numbers of lesions } \\
\text { per leaf }\end{array}$ \\
\hline CnA & $128.6 \pm 28.5$ & $663 \pm 56$ \\
205 C3 & $58.4 \pm 13.7$ & $487 \pm 28$ \\
$\Delta$ ctb1-T1 & $4.5 \pm 0.8$ & $64 \pm 31$ \\
$\Delta$ ctb1-T6 & $4.3 \pm 0.5$ & $57 \pm 18$ \\
$\Delta$ ctb1-T7 & $5.8 \pm 1.2$ & $16 \pm 8$ \\
$\Delta$ ctb1-T11 & $4.9 \pm 1.5$ & $24 \pm 9$ \\
$\Delta$ ctb1-T18 & $6.8 \pm 2.1$ & $78 \pm 21$ \\
$\Delta$ ctb1-T19 & $4.6 \pm 0.4$ & $15 \pm 7$ \\
$\Delta$ ctb1-T26 & $4.4 \pm 0.2$ & $63 \pm 16$ \\
$\Delta$ ctb1-T31 & $4.1 \pm 1.1$ & $40 \pm 11$ \\
$\Delta$ ctb1-T32 & $3.6 \pm 0.8$ & $528 \pm 72$ \\
T1R18 & $136.1 \pm 12.7$ & $617 \pm 54$ \\
T19R5 & $122.9 \pm 23.6$ & $\ldots$ \\
Control & $4.7 \pm 1.8$ &
\end{tabular}

${ }^{a}$ Fungal mycelium of $C$. nicotianae strains was grown on thin potato dextrose agar (PDA) plates. Cercosporin was extracted with $5 \mathrm{~N} \mathrm{KOH}$ and quantified by measuring absorbance of the extract at $480 \mathrm{~nm}$. Control consisted of five PDA plugs extracted with $5 \mathrm{~N} \mathrm{KOH}$. Data are the mean of two different experiments with five replicates of each isolate. Data shown \pm standard error of the mean.

${ }^{b}$ Fungal conidia were sprayed onto leaves of 6- to 8-week-old tobacco plants (cv. Burley 21) and necrotic lesions were scored 21 days after inoculation. Values are the mean of at least three different experiments with three leaves per isolate. Data shown \pm standard error of the mean. 
equivalent to those of the controls, were obtained for all $c t b 1$ disruption mutants. To determine whether the low calculated amounts were due to the presence of cercosporin or to small amounts of interfering pigments in the crude extract, fungal mycelium also was extracted with ethyl acetate and the extract separated by thin-layer chromatography (TLC). No cercosporin was identified by TLC from any of the eight $c t b 1$-null mutants, indicating that disruption of the $C T B 1$ gene completely blocks cercosporin biosynthesis (Fig. 4). As an additional control for these experiments, a mutant (205C3) deficient in a zinc cluster transcription factor, CRG1, which is required for normal cercosporin resistance and production, was tested. Cercosporin production by this mutant was approximately half that of the wild type (Table 1), consistent with previous findings with this mutant (Chung et al. 2003b).

Beyond abolishing cercosporin production, no other phenotypic characteristics were observed among the $c t b 1$-null mutants. All $c t b 1$-null mutants exhibited normal conidiation on V8 juice agar. In addition, when tested for cercosporin resistance by growth, in the presence of exogenous cercosporin, they retained normal growth compared with the wild-type strain (data not shown).

\section{Expression of the $C T B 1$ gene.}

The production of cercosporin by Cercospora spp. in vitro is strongly influenced by medium composition and environmental factors (Jenns et al. 1989). To determine whether CTB1 transcript accumulation in $C$. nicotianae is correlated with cercosporin production and regulated by light and medium composition, wild-type $C$. nicotianae cultures were grown in either continuous light or continuous darkness on PDA or complete medium (CM). Total RNA extracted from these cultures was hybridized to a $C T B 1$-specific probe. Northern blot analysis indicated that the $6.8-\mathrm{kb} C T B 1$ gene transcript accumulates to high levels when the fungus was grown in the PDA medium under continuous light (Fig. 5A), a condition also supporting high amounts of cercosporin accumulation (Fig. 5B). In contrast, $C T B 1$ transcript was undetectable and little cercosporin accumulated when the fungus was grown in continuous darkness. The accumulation of CTBI transcript was markedly reduced when the fungus was grown in the $\mathrm{CM}$ and cercosporin biosynthesis was suppressed in culture grown under continuous light (Fig. 5).

\section{Cercosporin as a virulence factor.}

To test whether production of cercosporin is required for fungal virulence and symptom development, the wild type, two CTB1-complemented strains (T1R18 and T19R5), and nine $c t b 1$-null mutants ( $\Delta$ ctb-T1, T6, T7, T11, T18, T19, T26, $\mathrm{T} 31$, and T32) were inoculated onto tobacco leaves. Inocula-

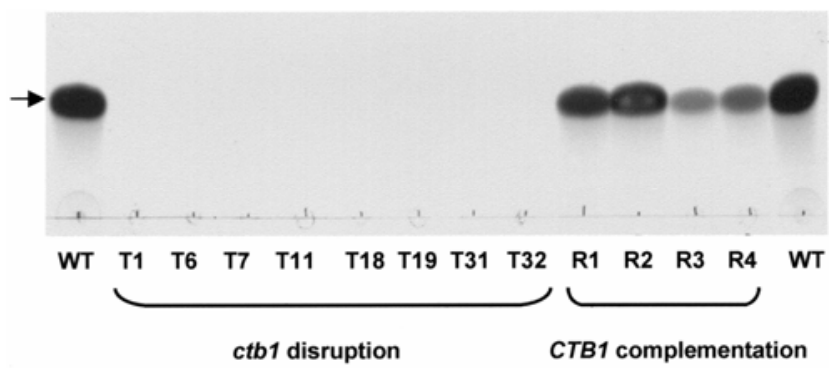

Fig. 4. Thin-layer chromatography analysis of cercosporin production by the wild type (WT), ctb1-null mutants (T), and CTB1-complemented (R) strains of Cercospora nicotianae. Cercosporin was extracted from fungal mycelium with ethyl acetate, spotted onto a silica gel plate, and separated with ethyl acetate:hexane:methanol: $\mathrm{H}_{2} \mathrm{O}(6: 4: 1.5: 1$, vol/vol). Cercosporin (indicated by an arrowhead) was easily detected as a red pigment at $R f 0.55$. tion of tobacco leaves with the wild type and complemented strains resulted in numerous necrotic lesions (Table 1; Fig. 6A). The necrotic lesions and the surrounding yellow halos caused by the wild-type strain and complemented strains enlarged and often coalesced to form blight regions on the leaf. In comparison, $c t b 1$-null mutants produced significantly fewer lesions (Table 1; Fig. 6A). Lesions produced by ctb1null mutants generally remained small and did not coalesce. The $\operatorname{crg} 1$ transcription regulator-disruption mutant (205C3), which produced approximately half of the cercosporin produced by the wild type, resulted in a disease level intermediate between wild type and the $c t b 1$-disruption mutants (Table 1). Inoculation with the $205 \mathrm{C} 3$ strain resulted in 20 to $25 \%$ fewer lesions, and the size of lesions induced by $205 \mathrm{C} 3$ was much smaller compared with those of the wild type (Fig. 6A).

Infection assessed in a detached leaf assay indicated that the ctb1-null mutants exhibit lower virulence on both wounded and unwounded tobacco leaves (Fig. 6B). In general, chlorotic symptoms developed earlier on wounded leaves inoculated with either the wild type or the $c t b l$-null mutants. However, significant differences in the progression of lesions between the wild
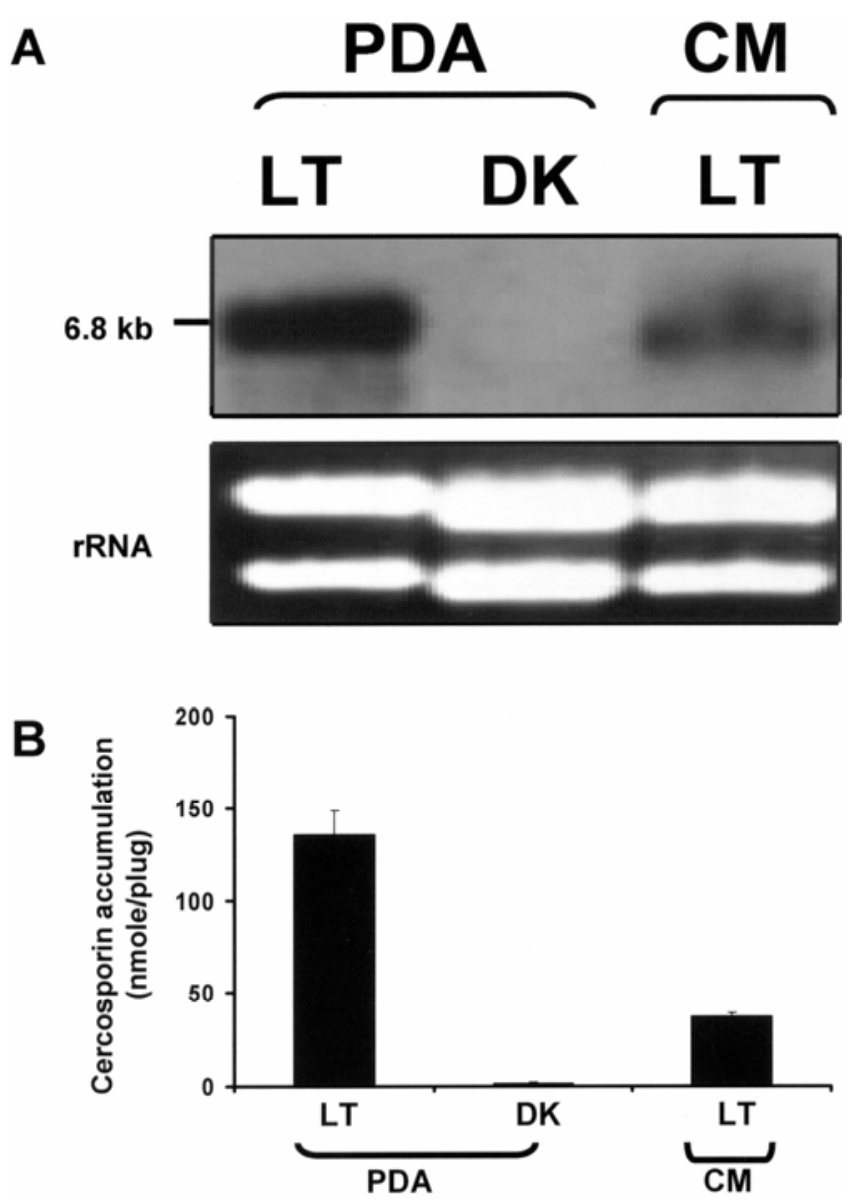

Fig. 5. Expression of the $C T B 1$ gene and production of cercosporin toxin by the wild-type Cercospora nicotianae. A, Northern blot analysis of CTB1 expression in $C$. nicotianae wild type grown on potato dextrose agar (PDA) or complete medium (CM) under continuous light (LT) or darkness (DK). Total fungal RNA was purified, electrophoresed in a formaldehydecontaining gel, blotted to membrane, and hybridized with a $C T B 1$-specific probe. Ethidium bromide-stained rRNA is shown to ensure equal loading of the samples. B, Production of cercosporin toxin by the wild-type $C$. nicotianae. The fungus was grown on PDA or CM plates under continuous light (LT) or darkness (DK) for 5 days, and cercosporin was extracted with $5 \mathrm{~N} \mathrm{KOH}$ and quantified by absorbance at $480 \mathrm{~nm}$. Data shown are the means and standard errors of three different experiments with five replicates of each treatment. 
type and the $c t b 1$-null mutants were noticed. The wild-type strain produced coalesced necrotic lesions with large yellow halos 2 weeks after inoculation on wounded leaves. In contrast, the $c t b 1$-null mutants incited fewer and smaller necrotic spots compared with those of the wild type on either unwounded or wounded leaves, and the lesions progressed slowly. Collectively, disruption of the CTB1 gene resulted in mutants that are completely devoid of cercosporin production and are markedly reduced in virulence. Complementation of $c t b 1$-null mutants with the full-length $C T B 1$ clone restored both cercosporin biosynthesis and full virulence to levels equivalent to those of the wild type. These data clearly indicate that cercosporin is an important virulence factor in C. nicotianae.

\section{DISCUSSION}

Based on structure and limited ${ }^{14} \mathrm{C}$-labeling experiments, the biosynthesis of the perylenequinone cercosporin has long been proposed to be via a polyketide pathway (Okubo et al. 1975). However, experimental documentation of this hypothesis has never been obtained. In this study, we isolated a fungal PKS gene, $C T B 1$, and determined its role in cercosporin biosynthesis in $C$. nicotianae. Disruption of the CTB1 gene with a hygromycin-resistance cassette yielded mutants that were defective in cercosporin production. Further, transformation of the CTB1 gene into $c t b 1$-null mutants restored cercosporin production. Thus, our results provide conclusive molecular evidence to support the hypothesis that cercosporin is biosynthesized via a polyketide pathway.

The predicted amino acid sequences of the CTB1 translation product showed strong similarity to many fungal type I PKSs. The CTB1 exhibited strong similarities to the Aspergillus fumigatus ALB1 protein (accession number AAC39471) involved in conidial pigment biosynthesis, the Emericella nidulans STCA-EMENI protein (accession number Q12397) involved in sterigmatocystin biosynthesis, and the E. nidulans WA protein (accession number Q03149) involved in conidial yellow pigment biosynthesis (Brown et al. 1996; Mayorga and Timberlake 1992; Tsai et al. 1998). CTB1 also displayed strong similarity to A. parasiticus PKSL1 protein (accession number Q12053) involved in aflatoxin biosynthesis (Feng and Leonard 1995).

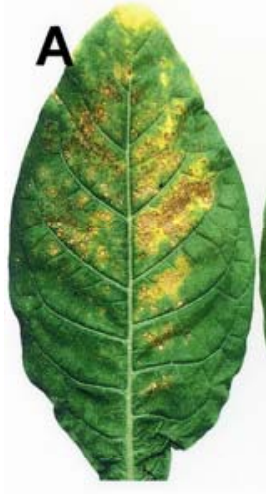

WT

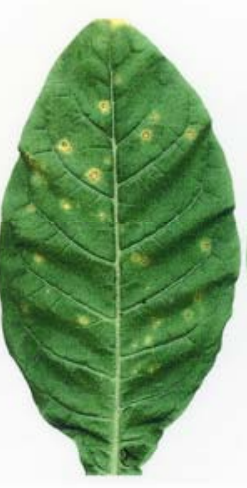

$\Delta$ ctb1-T19

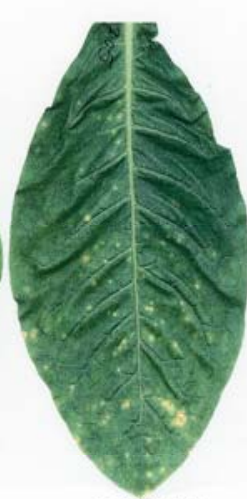

$205 C 3$

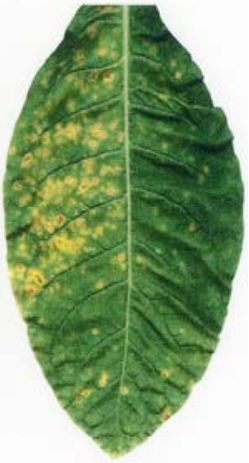

T19R-5

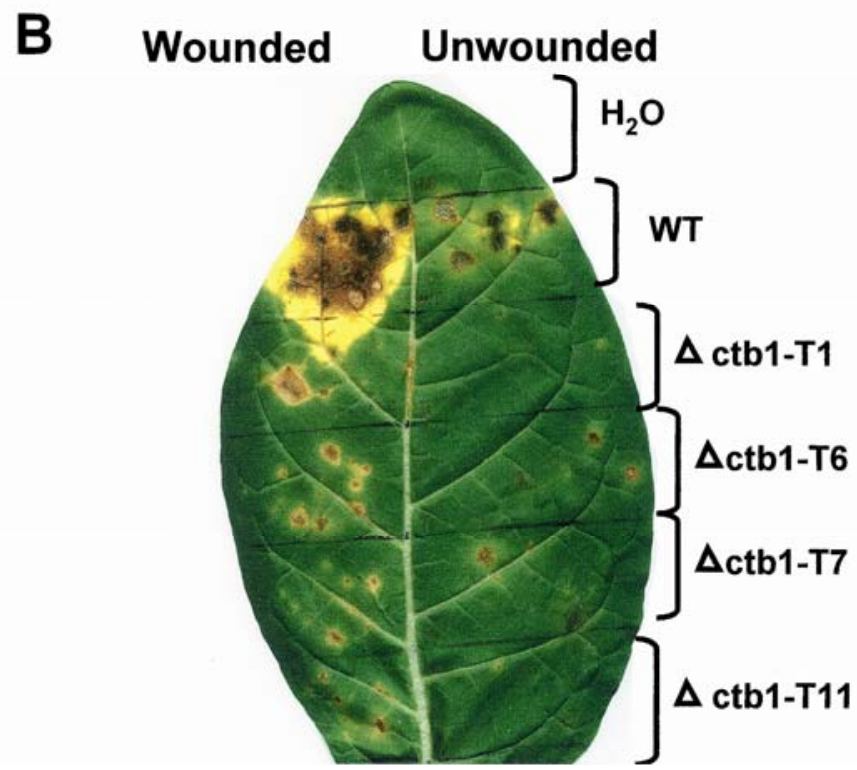

Fig. 6. Requirement of CTB1 for disease severity on tobacco leaves infected with Cercospora nicotianae. A, Pathogenicity assay using the whole tobacco plants. The wild type (WT), ctb1-null mutant (Actb1-T19), CTB1-complemented strain (T19R-5), and crg1-null mutant (205C3) were grown on V8 juice agar for 7 days at $20^{\circ} \mathrm{C}$ under continuous light. Conidia were harvested and sprayed onto leaves of 6- to 8-week-old tobacco cv. Burley 21 . Disease severity, evident by lesions with necrotic centers and yellow halos, was documented 14 to 21 days post inoculation. B, Pathogenicity assay on detached tobacco leaves. Conidial suspensions prepared from the wild type (WT) and ctb1-null mutants ( $\Delta$ ctb1-T1, T6, T7, and T11) of $C$. nicotianae were rubbed onto wounded and unwounded (separated by midrib) tobacco leaves with cotton swabs. The inoculated leaves then were incubated in a moist chamber under fluorescent light for 2 to 3 weeks. 
Given that cercosporin is a polyketide, the $C T B 1$ product likely functions as do fatty acid synthases, catalyzing the condensation of two-carbon units in each biosynthetic cycle using acetate and malonate to form polyketomethylene backbones (Okubo et al. 1975). CTB1 consisted of five catalytic domains, including a KS, an AT, a TE/CYC, and two ACP domains (KSAT-ACP-ACP-TE) required for elongation and condensation of cyclic cercosporin. The five catalytic domains were distributed at comparable distances along the protein with similar length to other fungal polyketides (data not shown). CTB1 contained a conserved cysteine residue in the $\mathrm{KS}$ domain and conserved serine residues in the AT, ACP, and TE domains similar to many fungal PKSs (Yang et al. 1996). The two ACP domains likely are involved in carbon chain elongation by serving as binding sites for phosphopantotheine (Katz and Donadio 1993). It is uncertain why CTB1 contains two ACP motifs. However, studies by using site-directed mutagenesis of the active serine residue in two ACP motifs of the E. nidulans WA protein revealed that either of them is sufficient to allow PKS function (Fujii et al. 2001). The AT domain likely is required for transferring the acetate unit from acetyl-CoA to the pantotheine arm of the ACP motif, whereas the KS domain is involved in condensation of the malonate unit with acetate by decarboxylation (Katz and Donadio 1993). The chain elongation and condensation steps are repeated until the chain reaches full length and the mature chain is released by the action of TE, followed by methylation. The $\beta$-keto group in the polyketide chain presumably remains unchanged or reduced to a hydroxyl because CTB1 lacks KR, DH, and ER domains.

The catalytic serine residue in the TE domain of CTB1 may likely be involved in cyclization of the aromatic ring of cercosporin as demonstrated for the WA protein that catalyzes naphthopyrone biosynthesis (Fujii et al. 2001). Deletion and sitedirected mutagenesis of WA also revealed the important role in cyclization of the histidine residue in the C-terminus of WA. CTB1 also contained a conserved histidine residue in the $\mathrm{C}$ terminus, suggestive of a similar function for ring closure. Furthermore, CTB1 shares with many fungal type-I PKSs a conserved sequence (LFGDQ) in the N-terminus (data not shown). Deletion of these five amino acids in the N-terminus of WA eliminates the polyketide synthase activity in E. nidulans (Fujii et al. 2001).

Cercosporin contains a bilateral symmetrical structure; therefore, cercosporin likely is formed from fusing two identical polyketomethylene chains in the final step (Okubo et al. 1975). Except for CTB1, proteins that catalyze subsequent reactions involving decarboxylation, methylation, and hydroxylation also must be required for cercosporin biosynthesis. As has been shown with many fungal secondary metabolites (Keller and Hohn 1997), genes involved in the biosynthetic pathway of cercosporin may be clustered in Cercospora spp. Recently, sequence analysis of the surrounding DNA fragments of the CTB1 gene identified several putative ORFs with amino acid similarities to $O$-methyltransferases, oxidoreductases, hydroxylases, major facilitator superfamily transporters, and transcriptional activators (K.-R. Chung, K. L. Dekkers, and M. Choquer, unpublished data). Whether or not these genes that cluster with $C T B 1$ are also involved in cercosporin biosynthesis awaits further analysis.

Light is not only crucial for cercosporin toxicity, but also is required for the initiation of cercosporin biosynthesis (Ehrenshaft and Upchurch 1991, 1993). Production of cercosporin is also markedly affected by medium composition. For example, PDA medium supports high amounts of cercosporin accumulation in culture, whereas CM significantly reduced its biosynthesis (Jenns et al. 1989). Expression of the CTB1 gene, as assessed by Northern blot hybridization, revealed that the
CTB1 gene transcript is highly inducible by light and in mycelium grown on PDA. The light- or medium-induced cercosporin production was correlated with light- or mediumenhanced $C T B 1$ gene transcript accumulation, suggesting that Cercospora spp. synthesize cercosporin toxin in response to light or other environmental cues by activation of $C T B 1$ gene expression. $C T B 1$ gene activation by environmental cues likely may involve calcium or calmodulin signaling because cercosporin production is significantly reduced by various calcium or calmodulin inhibitors in C. nicotianae (Chung 2003). A preliminary study also indicated that the $C T B 1$ gene transcript was markedly reduced when the fungus was grown in media containing calcium or calmodulin inhibitors (K. L. Dekkers, personal communication).

One of the most significant findings of this study is the confirmation that cercosporin is required for full virulence in C. nicotianae. For many years, cercosporin has been suggested to be crucial for fungal pathogenesis by Cercospora spp. (Daub and Ehrenshaft 2000). The earliest studies correlated toxin-deficient UV-generated mutants of C. kikuchii with reduced virulence on soybean (Upchurch et al. 1991). Subsequently, Upchurch and co-workers isolated from C. kikuchii a gene for a cercosporin facilitator protein that is hypothesized to be the cercosporin transporter (Callahan et al. 1999). Disruption of the CFP gene severely attenuated disease symptoms on soybean and reduced, but did not eliminate, cercosporin production. Mutants of $C$. zeae-maydis disrupted in the MAP kinase gene $C Z K 3$, which is upregulated during cercosporin production, were completely defective in cercosporin production and also significantly reduced in virulence; however, these mutants also were defective in conidiation, preventing definitive conclusions for a role of cercosporin in Cercospora pathogenesis (Shim and Dunkle 2003). In our present study, we were able to recover mutants completely deficient in cercosporin production by specifically disrupting the initial biosynthetic gene in the pathway. All ctbl disruption mutants were significantly reduced in virulence when inoculated on tobacco. Moreover, genetic complementation of ctb1-null mutants with the full-length $C T B 1$ gene fully restored normal production of cercosporin in culture as well as normal disease symptoms. Taken together, these results conclusively demonstrated that cercosporin is a crucial factor in pathogenesis of tobacco by $C$. nicotianae. The study suggests that strategies that interfere with the toxicity of cercosporin will be useful in reduction of disease incidence caused by Cercospora spp.

\section{MATERIALS AND METHODS}

\section{Fungal strains, media, cultural conditions, and toxin quantification.}

C. nicotianae wild-type strain ATCC18366, the cercosporin toxin biosynthesis mutant (ctb1), and other strains were maintained on CM (Jenns et al. 1989). For protoplast preparation, fungal isolates were grown in $50 \mathrm{ml}$ of potato dextrose broth (PDB; Difco Laboratories, Detroit) with shaking for 4 to 5 days in the dark. Fungal mycelium was ground in a sterile blender, mixed with $200 \mathrm{ml}$ of fresh medium, and grown for an additional $16 \mathrm{~h}$ at $28^{\circ} \mathrm{C}$ in the dark. For DNA and RNA purification, fungal strains were grown on CM or PDA with a layer of cellophane (cellulose; Paper Mart, Los Angeles). Fungal mycelium can be separated easily from cellophane without contamination of agar. Screening of cercosporin-deficient mutants was conducted on thin PDA (Difco Laboratories) plates as described previously (Chung et al. 2003c). Quantitative assays for cercosporin production were conducted by extracting agar plugs cut from mycelial cultures with $5 \mathrm{~N} \mathrm{KOH}$ or with 
ethyl acetate. Spectrophotometric quantification and TLC were conducted as described (Chung 2003; Fore et al. 1988). KOH extracts were quantified by measuring absorbance at $480 \mathrm{~nm}$. TLC utilized TLC plates with a 60 F254 fluorescent silica gel ( 5 by $20 \mathrm{~cm}$; Selecto Scientific Inc., Suwanee, GA) and a solvent system containing ethyl acetate/hexane/methanol/ $\mathrm{H}_{2} \mathrm{O}$ (6:4:1.5:1, vol/vol). Assays for cercosporin sensitivity were performed on $\mathrm{CM}$ amended with $10 \mu \mathrm{M}$ cercosporin as described by Jenns and Daub (1995). Pure cercosporin was purchased from Sigma-Aldrich (St. Louis) and dissolved in acetone to make a $10 \mathrm{mM}$ stock solution.

\section{Cloning \\ of the full-length cercosporin biosynthesis (CTB1) gene.}

The full-length $C T B 1$ gene encoding a fungal PKS was obtained with two inverse primers derived from the rescued plasmid pCTB1 (Fig. 1) (Chung et al. 2003c). Briefly, C. nicotianae genomic DNA was digested with EcoRV and selfligated with a T4 DNA ligase. An 8.0-kb fragment containing the $5^{\prime}$ end $C T B 1$ was amplified with primers pks-5 (5'-ttgac taggaggacggcag-3') and pks-6 (5'-ttgatggacgcaaaggtctg- $\left.3^{\prime}\right)$ with Elongase enzyme mix (Invitrogen, Carlsbad, CA) and cloned into the pGEM-T easy vector (Promega Corp., Madison, WI, U.S.A.) to yield pPKS-8 for sequence analysis (Fig. 1). A $3.7-\mathrm{kb}$ fragment containing the $3^{\prime} C T B 1$ terminus then was amplified with inverse primers $1209 \mathrm{R}\left(5^{\prime}\right.$-gtgtgtcggcactg atagcc- $\left.3^{\prime}\right)$ and $1209 \mathrm{~L}\left(5^{\prime}\right.$-ctgacctcgttcgcgttcat- $\left.3^{\prime}\right)$ from $\mathrm{NcoI}-$ digested and self-ligated genomic DNA pools, and cloned into the pGEM-T vector to yield pCNA1 for sequence analysis (Fig. 1). DNA sequence analysis with the ABI Prism Bigdye primer cycler sequencing kit (Applied Biosystems, Foster City, CA, U.S.A.) was conducted at the Integrated Biotechnology Laboratory, University of Georgia (Athens). Database searches and comparisons were performed at the National Center of Biotechnology Information using the BLAST network service (Altschul et al. 1997) and ExPASy Molecular Biology servers.

\section{Fungal transformation, gene disruption, and genetic complementation.}

Fungal protoplasts were prepared and transformed as described previously (Chung et al. 2002). To create a CTB1 disruption vector, a $7-\mathrm{kb}$ fragment containing the whole $C T B 1$ gene was amplified from genomic DNA of $C$. nicotianae with two primers, P1 (5'-gacatccegcaccatcgaag- $\left.3^{\prime}\right)$ and P12 (5'-gctgcgaatgcgagcgctag-3'), by an Expand High Fidelity PCR system (Roche Applied Science, Indianapolis, IN, U.S.A.), and cloned into pGEM-T vector to result in pCTB112. A 2.9-kb KpnI/EcoRV fragment in pCTB112 then was replaced with a 1.6-kb hygromycin phosphotransferase B gene $(H Y G)$ cassette from pUCATPH (Lu et al. 1994) to yield pCTB115. To facilitate $C T B 1$ gene replacement, a splitmarker recombination procedure (Catlett et al. 2003; Fairhead et al. 1996) was adapted for $C$. nicotianae. Split- $H Y G$ marker fused with truncated $C T B 1$ was generated by PCR with two sets of primers from pCTB115 (Fig. 3A). A 2.6-kb fragment containing $5^{\prime}$ CTB1 fused with $3^{\prime}$ HYG was amplified with primers $0315 \mathrm{~L}$ (5'-ggcagtctcacagctcttgag- $\left.3^{\prime}\right)$ and hyg3 ( $5^{\prime}$-ggatgcctccgctcgaagta-3'). A $2.4-\mathrm{kb}$ fragment containing 5' $H Y G$ fused with $3^{\prime} C T B 1$ was amplified with primers 0315R (5'-ccgggtaagaggtgcagtttcg- $\left.3^{\prime}\right)$ and hyg4 (5'-cgttgcaa gaactgcctgaa- $\left.3^{\prime}\right)$. PCR fragments, overlapping $450 \mathrm{bp}$ at the $H Y G$ region, were purified with an Ultraclean PCR clean-up kit (Mo Bio Laboratories, Carlsbad, CA, U.S.A.) and directly transformed into the $C$. nicotianae wild-type strain. For genetic complementation, an $8.2-\mathrm{kb}$ fragment was amplified with primers ctb1-NotI (5'-gttgtgtttccgcggccgcttgtacgc-3') and
ctb1-SpeI (5'-gtcatgataactagtcggcca-3') containing restriction enzyme sites NotI and SpeI, respectively. After digestion with NotI and SpeI, the fragment was cloned into pCB1532 containing a sulfonylurea-resistance gene cassette (Sweigard et al. 1997) to yield pCTB117. The pCTB117 was used to complement the $c t b 1$-null mutants $\Delta$ ctb-T1 and T19. Transformants were selected in medium containing hygromycin (Roche Applied Science) at $250 \mu \mathrm{g} / \mathrm{ml}$ or sulfonylurea (chlorimuron ethyl; Chem Service, West Chester, PA, U.S.A.) at 5 $\mu \mathrm{g} / \mathrm{ml}$ as appropriate and tested for cercosporin production on thin PDA plates as described previously (Chung 2003).

\section{Southern and Northern blot analyses.}

Total fungal DNA was isolated with a DNeasy Plant Mini kit (Qiagen, Valencia, CA, U.S.A.). Total fungal RNA was extracted with a TRIZOL RNA Isolator kit (Invitrogen). Standard procedures were used for endonuclease digestion of DNA, electrophoresis, and Southern and Northern blotting (Sambrook and Russell 2001). The hybridization probes were labeled with digoxigenin-11-dUTP (Roche Applied Science) by PCR as described previously (Chung et al. 2003a). Posthybridization washing and immunological detection of the probe using a CSPD lumigenic substrate for alkaline phosphatase were conducted according to the manufacturer's recommendations (Roche Applied Science). Oligonucleotide primers for PCR were synthesized by Integrated DNA Technologies (Coralville, IA, U.S.A.).

\section{Reverse-transcriptase PCR, and cDNA isolation.}

The poly $\left(\mathrm{A}^{+}\right)$mRNA was isolated with an Oligotex kit (Qiagen). The first strand cDNA was synthesized from poly $\left(\mathrm{A}^{+}\right)$mRNA with a Sensiscript reverse transcriptase (Qiagen) and oligo $\mathrm{dT}_{30}$ primer. Four overlapping cDNA fragments were subsequently amplified with an AccuPrime Taq DNA polymerase (Invitrogen) and CTBI gene-specific primers, and the fragments were cloned into the pGEM-T easy vector (Promega Corp.) for sequence analysis (Fig. 1).

\section{Fungal pathogenicity test.}

Fungal inoculations initially were performed in greenhouse tests on 6- to 8-week-old tobacco plants, cv. Burley 21. Cultures of fungal strains were induced to produce conidia by culturing them on V8-juice-agar medium as described by Jenns and Daub (1995). Conidia were harvested after 7 days of growth at $20^{\circ} \mathrm{C}$ under continuous light. The concentration of conidia was adjusted to approximately $5 \times 10^{4}$ conidia $/ \mathrm{ml}$ with the aid of a hemacytometer, and then sprayed onto tobacco leaves with a SpraTool sprayer (Crown, Woodstock, IL, U.S.A.). Inoculated plants were covered individually with plastic bags, kept shaded for 4 days, and then transferred to greenhouse benches. The number and size of lesions from at least three leaves of each plant were determined 14 to 21 days after inoculation. To minimize a potential release of genetically modified fungi, pathogenicity assays also were conducted on detached tobacco leaves. Briefly, conidial suspensions were rubbed onto the detached leaves with a cotton swab, and the inoculated leaves were kept in a moist chamber under fluorescent light at room temperature for 2 to 3 weeks. Leaves were wounded with sterile needles prior to inoculation. The experiments were repeated three times with at least three replicates.

\section{ACKNOWLEDGMENTS}

This research was supported by the Florida Agricultural Experiment Station and approved for publication as Journal Series Number R-10534. We thank D. J. Lewandowski for his suggestions in propagating tobacco 
plants in the greenhouse; and S. Gowda, J. K. Burns, and two anonymous reviewers for their helpful comments.

\section{LITERATURE CITED}

Altschul, S. F., Madden, T. L., Schaffer, A. A., Zhang, J., Zhang, Z., Miller, W., and Lipman, D. J. 1997. Gapped BLAST and PSI-BLAST: A new generation of protein database search programs. Nucleic Acids Res. 25:3389-3402.

Balis, C., and Payne, M. G. 1971. Triglycerides and cercosporin from Cercospora beticola: Fungal growth and cercosporin production. Phytopathology 61:1477-1484.

Brown, D. W., Yu, J.-H., Kelkar, H. S., Fernandes, M., Nesbitt, T. C., Keller, N. P., Adams, T. H., and Leonard, T. J. 1996. Twenty-five coregulated transcripts define a sterigmatocystin gene cluster in Aspergillus nidulans. Proc. Natl. Acad. Sci. U.S.A. 93:1418-1422.

Callahan, T. M., Rose, M. S., Meade, M. J., Ehrenshaft, M., and Upchurch, R. G. 1999. CFP, the putative cercosporin transporter of Cercospora kikuchii, is required for wild type cercosporin production, resistance, and virulence on soybean. Mol. Plant-Microbe Interact. 12:901-910

Calpouzos, L., and Stalknecht, G. F. 1967. Symptoms of Cercospora leaf spot of sugar beets influenced by light intensity. Phytopathology 57:799-800

Catlett, N. L., Lee, B.-N., Yoder, O. C., and Turgeon, B. G. 2003. Splitmarker recombination for efficient targeted deletion of fungal genes. Fungal Genet. Newsl. 50:9-11.

Chung, K.-R. 2003. Involvement of calcium/calmodulin signaling in cercosporin toxin biosynthesis by Cercospora nicotianae. Appl. Environ. Microbiol. 69:1187-1196.

Chung, K.-R., Shilts, T., Li, W., and Timmer, L. W. 2002. Engineering a genetic transformation system for Colletotrichum acutatum, the causal fungus of lime anthracnose and postbloom fruit drop. FEMS (Fed. Eur. Microbiol. Soc.) Microbiol. Lett. 213:33-39.

Chung, K.-R., Daub, M. E., and Ehrenshaft, M. 2003a. Expression of cercosporin toxin resistance gene $(C R G 1)$ as a dicistronic unit with a functionally unrelated gene in the phytopathogenic fungus Cercospora nicotianae. Curr. Genet. 43:415-424.

Chung, K.-R., Daub, M. E., Kuchler, K., and Schüller, C. 2003b. The $C R G 1$ gene required for resistance to the singlet oxygen-generating cercosporin toxin in Cercospora nicotianae encodes a putative fungal transcription factor. Biochem. Biophys. Res. Commun. 302:302-310.

Chung, K.-R., Ehrenshaft, M., Wetzel, D. K., and Daub, M. E. 2003c. Cercosporin toxin deficient mutants by plasmid tagging in the asexual fungus Cercospora nicotianae. Mol. Genet. Genomics 270:103-113.

Daub, M. E. 1982a. Peroxidation of tobacco membrane lipids by the photosensitizing toxin, cercosporin. Plant Physiol. 69:1361-1364.

Daub, M. E. 1982b. Cercosporin, a photosensitizing toxin from Cercospora species. Phytopathology 72:370-374.

Daub, M. E., and Ehrenshaft, M. 2000. The photoactivated Cercospora toxin cercosporin: Contributions to plant disease and fundamental biology. Annu. Rev. Phytopathol. 38:461-490.

Daub, M. E., and Hangarter, R. P. 1983. Production of singlet oxygen and superoxide by the fungal toxin, cercosporin. Plant Physiol. 73:855-857.

Daub, M. E., Leisman, G. B., Clark, R. A., and Bowden, E. F. 1992 Reduced detoxification as a mechanism of fungal resistance to singletoxygen-generating photosensitizers. Proc. Natl. Acad. Sci. U.S.A. 89:9588-9592.

Daub, M. E., Li, M., Bilski, P., and Chignell, C. F. 2000. Dihydrocercosporin singlet oxygen production and subcellular localization: A possible defense against cercosporin phototoxicity in Cercospora. Photochem. Photobiol. 71:135-140.

Ehrenshaft, M., and Upchurch, R. G. 1991. Isolation of light-enhanced cDNA clones of Cercospora kikuchii. Appl. Environ. Microbiol. 57:2671-2676.

Ehrenshaft, M., and Upchurch, R. G. 1993. Host protein(s) induces accumulation of the toxin cercosporin and mRNA in a phytopathogenic strain of Cercospora kikuchii. Physiol. Plant Pathol. 79:157-164.

Ehrenshaft, M., Jenns, A. J., Chung, K.-R., and Daub, M. E. 1998. SOR1, a gene required for photosensitizer and singlet oxygen resistance in Cercospora fungi, is highly conserved in divergent organisms. Mol. Cell 1:603-609.

Ehrenshaft, M., Bilski, P., Li, M., Chignell, C. F., and Daub, M. E. 1999. A highly conserved sequence is a novel gene involved in de novo vitamin B6 biosynthesis. Proc. Natl. Acad. Sci. U.S.A. 96:9374-9378.
Fairhead, C., Llorente, B., Denis, F., Soler, M., and Dujon, B. 1996. New vectors for combinatorial deletions in yeast chromosomes and for gaprepair cloning using 'split-marker' recombination. Yeast 12:1439-1457.

Fajola, A. O. 1978. Cercosporin, a phytotoxin from Cercospora species. Physiol. Plant Pathol. 13:157-164.

Feng, G. H., and Leonard, T. J. 1995. Characterization of the polyketide synthase gene ( $p k s L 1)$ required for aflatoxin biosynthesis in Aspergillus parasiticus. J. Bacteriol. 177:6246-6254.

Fore, S. A. Daub, M. E., and Beute, M. K. 1988. Phytotoxic substances produced by some isolates of Cercospora arachidicola are not cercosporin. Phytopathology 78:1082-1086.

Fujii, I., Mori, Y., Watanabe, A., Kubo, Y., Tsuji, G., and Ebizuka, Y. 2001. Identification of claisen cyclase domain in fungal polyketide synthase WA, a naphthopyrone synthase of Aspergillus nidulans. Chem. Biol. 8:189-197.

Jenns, A. E., and Daub, M. E. 1995. Characterization of mutants of Cercospora nicotianae sensitive to the toxin cercosporin. Phytopathology 85:906-912.

Jenns, A. E., Daub, M. E., and Upchurch, R. G. 1989. Regulation of cercosporin accumulation in culture by medium and temperature manipulation. Phytopathology 79:213-219.

Katz, L., and Donadio, S. 1993. Polyketide synthesis: Prospects for hybrid antibiotics. Annu. Rev. Microbiol. 47:875-912.

Keller, N. P., and Hohn, T. M. 1997. Metabolic pathway gene clusters in filamentous fungi. Fungal Genet. Biol. 21:17-29.

Kozak, M. 1984. Compilation and analysis of sequences upstream from the translational start site in eukaryotic mRNAs. Nucleic Acids Res. 12:857-872.

Kuyama, S., and Tamura, T. 1957. Cercosporin. A pigment of Cercospora kikuchii Matsumoto et Tomoyasu. I. Cultivation of fungus, isolation and purification of pigment. J. Am. Chem. Soc. 79:5725-5726.

Lu, S., Lyngholm, L., Yang, G., Bronson, C., Yoder, O. C., and Turgeon, B. G. 1994. Tagged mutations at the Tox1 locus of Cochliobolus heterostrophus by restriction enzyme-mediated integration. Proc. Natl. Acad. Sci. U.S.A. 91:12649-12653.

Macri, F., and Vianello, A. 1979. Photodynamic activity of cercosporin on plant tissues. Plant Cell Environ. 2:267-271.

Mayorga, M. E., and Timberlake, W. E. 1992. The developmentally regulated Aspergillus nidulans $w A$ gene encodes a polypeptide homologous to polyketide and fatty acid synthases. Mol. Gen. Genet. 235:205-212.

Okubo, A., Yamazak, S., and Fuwa, K. 1975. Biosynthesis of cercosporin. Agric. Biol. Chem. 39:1173-1175.

Radford, A., and Parish, J. H. 1997. The genome and genes of Neurospora crassa. Fungal Genet. Biol. 21:258-266.

Sambrook, J., and Russell, D. W. 2001. Molecular Cloning: A Laboratory Manual. 3rd ed. Cold Spring Harbor Laboratory Press, Cold Spring Harbor, NY, U.S.A

Shim, W.-B., and Dunkle, L. D. 2003. CZK3, a MAP kinase kinase kinase homolog in Cercospora zeae-maydis, regulates cercosporin biosynthesis, fungal development, and pathogenesis. Mol. Plant-Microbe Interact. 16:760-768.

Sweigard, J. A., Chumley, F. C., Carroll, A. M., Farrall, L., and Valent, B. 1997. A series of vectors for fungal transformation. Fungal Genet. Newsl. 44:52-53.

Tsai, H.-F., Chang, Y. C., Washburn, R. G., Wheeler, M. H., and KwonChung, K. L. 1998. The developmentally regulated alb1 gene of Aspergillus fumigatus: Its role in modulation of conidial morphology and virulence. J. Bacteriol. 180:3031-3038.

Upchurch, R. G., Walker, D. C., Rollins, J. A., Ehrenshaft, M., and Daub, M. E. 1991. Mutants of Cercospora kikuchii altered in cercosporin synthesis and pathogenicity. Appl. Environ. Microbiol. 57:29402945.

Yamazaki, S., Okube, A., Akiyama, Y., and Fuwa, K. 1975. Cercosporin, a novel photodynamic pigment isolated from Cercospora kikuchii. Agric. Biol. Chem. 39:287-288.

Yang, G., Rose, M., Turgeon, B. G., and Yoder, O. C. 1996. A polyketide synthase is required for fungal virulence and production of the polyketide T-toxin. Plant Cell 8:2139-2150.

\section{AUTHOR-RECOMMENDED INTERNET RESOURCES}

National Center of Biotechnology Information webpage: www.ncbi.nlm.nih.gov

The ExPASy (Expert Protein Analysis System) Molecular Biology proteomics server: au.expasy.org 